\title{
Extreme Tolerance of the Optic Nerve to Ionizing Radiation: A Case Report Revealing the Role of the Dose-Volume Effect
}

\author{
John R. Adler Jr. ${ }^{1}$, Judy Murovic ${ }^{2}$, Yaping Joyce Liao ${ }^{3}$, Lawrence M. Shuer ${ }^{4}$ \\ 1. Radiation Oncology, Stanford University Medical Center, Stanford, USA 2. Department of \\ Neurosurgery, Stanford University School of Medicine, Palo Alto, USA 3. Ophthalmology, Stanford \\ University Medical Center 4. Department of Neurosurgery, Stanford University Medical Center
}

$\square$ Corresponding author: John R. Adler Jr., jra@stanford.edu

Disclosures can be found in Additional Information at the end of the article

\section{Abstract}

Perhaps because of its innate, precarious blood supply, clinical experience has shown that the anterior visual pathways are especially sensitive to high doses of ionizing radiation. As a result, the risk of radiation injury to the optic nerves has engendered considerable angst in the setting of stereotactic radiosurgery (SRS). The general consensus has been that the likelihood of complications involving the optic nerves increases significantly at doses greater than 12 Gy [1]. However, this rule of thumb pays little credence to the near-universal concept within radiosurgery of the "volume effect". The current case demonstrates the importance of considering not just dose, but also volume, when assessing the risk of radiation-induced optic neuropathy. To the best of our knowledge, this case report documents the highest dose of ionizing radiation given to any portion of the anterior visual pathway without apparent injury.

Categories: Ophthalmology, Radiation Oncology, Neurosurgery

Keywords: radiosurgery, optic neuropathy, Stereotactic Radiosurgery, radiation injury, radiation tolerance

\section{Introduction}

Perhaps because of its innate, precarious blood supply, clinical experience has shown that the anterior visual pathways are especially sensitive to high doses of ionizing radiation. As a result, the risk of radiation injury to the optic nerves has engendered considerable angst in the setting of stereotactic radiosurgery (SRS). The general consensus has been that the likelihood of complications involving the optic nerves increases significantly at doses greater than 12 Gy [1]. However, this rule of thumb ignores the near-universal concept within radiosurgery of the "volume effect." The current case demonstrates the importance of considering not just dose, but also volume, when assessing the risk of radiation-induced optic neuropathy. To the best of our knowledge, this case report documents the highest dose of ionizing radiation given to any portion of the anterior visual pathway without apparent injury.

\section{Case Presentation}

Upon experiencing a painless loss of vision in her right eye (OD), a 27-year-old female underwent formal ophthalmologic evaluation, which revealed normal visual acuity in the left eye but light perception only visual acuity in the right eye as well as a right relative afferent 
pupillary defect and severe right optic atrophy. Computed tomography (CT) of the head with contrast revealed a small, enhancing mass at the orbital apex. Following biopsy and subtotal resection via a right frontal craniotomy, a diagnosis of optic nerve sheath Grade I meningioma (ONSM) was arrived at. Because the vision loss and optic atrophy in the OD was already severe, the goal of therapy was focused on preventing tumor progression, rather than visual recovery, especially with respect to intracranial extension.

After reviewing treatment options, the patient was offered and consented to undergo SRS tumor ablation. The radiosurgical procedure was performed in 1991 with a stereotactic framebased localization using CT-guidance and a 4 megavolt Varian linear accelerator, the technique for which has been previously reported [2]. Utilizing what was, at the time, a state-of-the-art treatment planning display for radiosurgery, Figure 1 depicts the calculated dosimetry for this case. Of significance, the tumor diameter was measured to be $6.7 \mathrm{~mm}$, and a $7.5 \mathrm{~mm}$ diameter, round collimator was used for the treatment. The planned dose was $2500 \mathrm{cGy}$, defined at the 80th percentile isodose, with a calculated intratumoral Dmax of 3125 cGy.

A

A Head Cross Section at Center (AP $=196.3 \quad$ RL $=141.5$ ) Malformation (dia $=6.7 \mathrm{~mm}$ )
Isodoses $10,20,30,40,50,60,70,80,90,100$

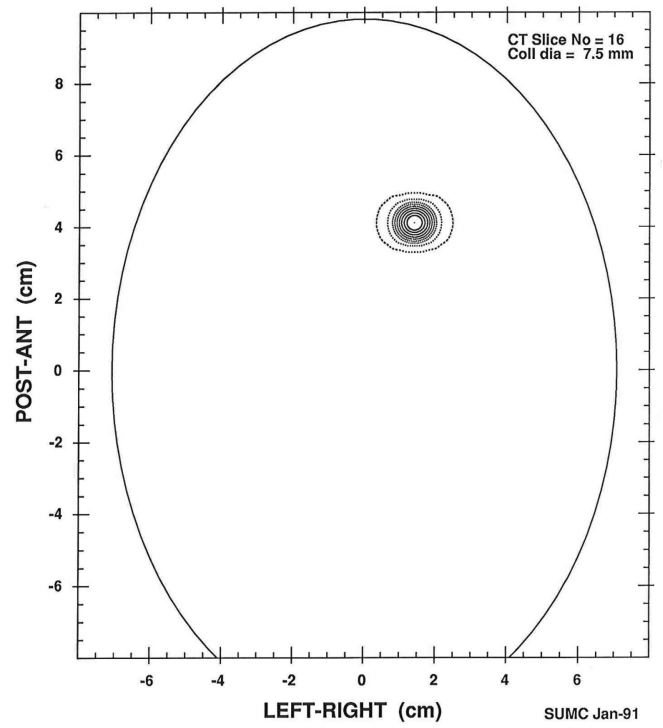

B $=$ Head Cross Section at Center (AP $=19.3$ RL 141.5 )

Head Cross Section at Isocenter

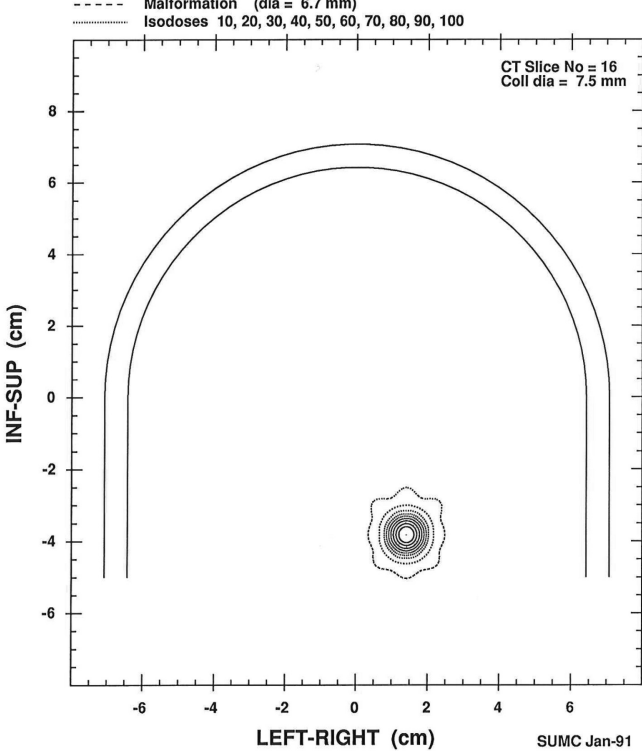

FIGURE 1: Image depicts the calculated dosimetry used in this case.

Both Figures 1A (axial) and 1B (coronal) show cross-sections through the $6.7 \mathrm{~mm}$ tumor, as well as the 10th-90th percentile isodose lines in $10 \%$ increments. The $y$-axis of Fig. $1 \mathrm{~A}$ corresponds to the tumor location in the posterior-anterior direction in centimeters $(\mathrm{cm})$ and in Fig. 1B, to the inferiorsuperior direction. The $\mathrm{x}$-axes in both Figs. $1 \mathrm{~A}$ and $1 \mathrm{~B}$ correspond to the tumor position in left-right directions, also in $\mathrm{cm}$.

Following radiosurgical ablation, the patient was followed regularly through ophthalmologic evaluations and contrast magnetic resonance imaging (MRI) of the orbits; the initial evaluations were done at six-month intervals and then gradually on an annual basis. Within a month of radiosurgery, the patient regained the ability to read large type in her OD, and in the successive years to follow, her vision subjectively improved. Although the earliest MRIs through the orbits have been destroyed, a contrast scan obtained 10 years after treatment was reported by a skilled neuroradiologist, who had access to the patient's older MRI of the orbits, as demonstrating "a stable, enhancing mass located in the right orbital apex". Figures $2 A, 2 B$ demonstrate two axial images from this scan, both with and without contrast, respectively. 


\section{Cureus}

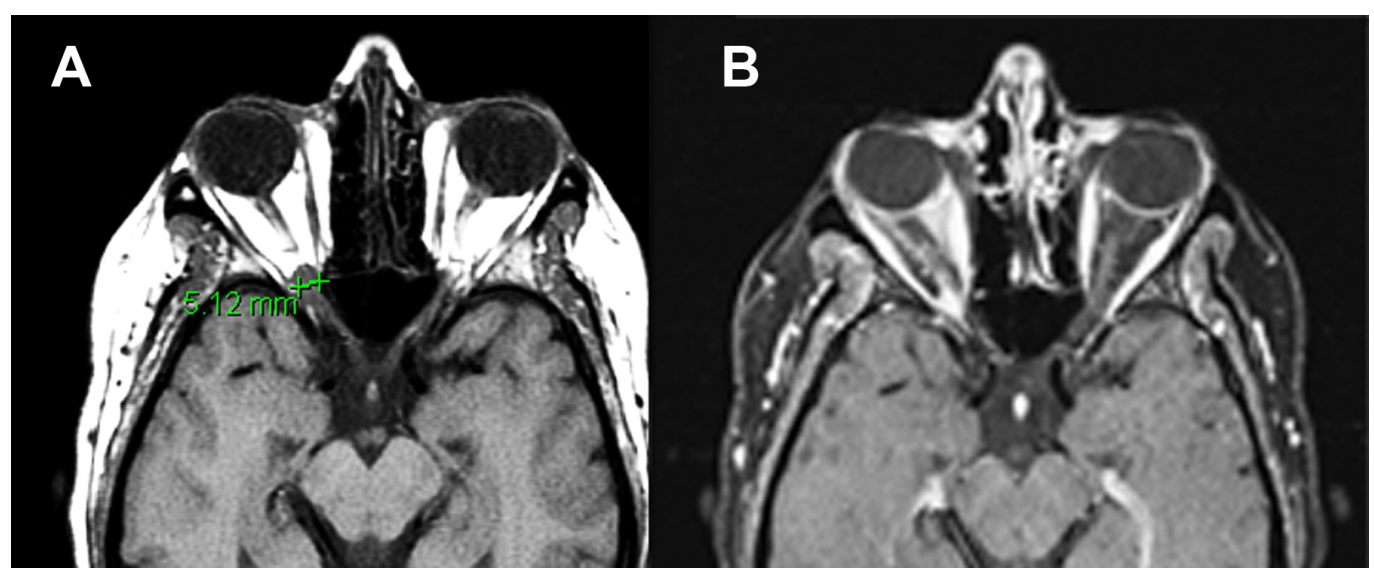

FIGURE 2: Magnetic resonance imaging (MRI) of the orbits of the right optic nerve sheath meningioma 10 years after

\section{radiosurgery.}

Figure 2A shows a T1-weighted, non-contrast, axial image through the right orbital apex showing a lesion which is isodense to the gray matter and measuring $5.12 \mathrm{~mm}$ in diameter. Figure 2B demonstrates a fat-suppressed, gadolinium-enhanced T1-weighted image through the same area. There is enhancement at the orbital apex consistent with the characteristic radiographic finding of an optic nerve sheath meningioma.

After being lost to follow-up for more than a decade, the patient underwent a formal ophthalmologic assessment and an MRI of the orbits 23 years after her initial pre-radiosurgery evaluation. Her best visual acuity in the right eye was 20/30, which was significantly improved compared with her prior light perception acuity before her radiosurgery. Her visual field study using static, automated perimetry (Humphrey visual field study, SITA-Standard) revealed relative sparing of the superior nasal better than temporal field in the right eye (Figure 3). A MRI of her orbits shows a stable enhancing lesion along the posterior optic nerve at the right orbital apex (Figure 4).
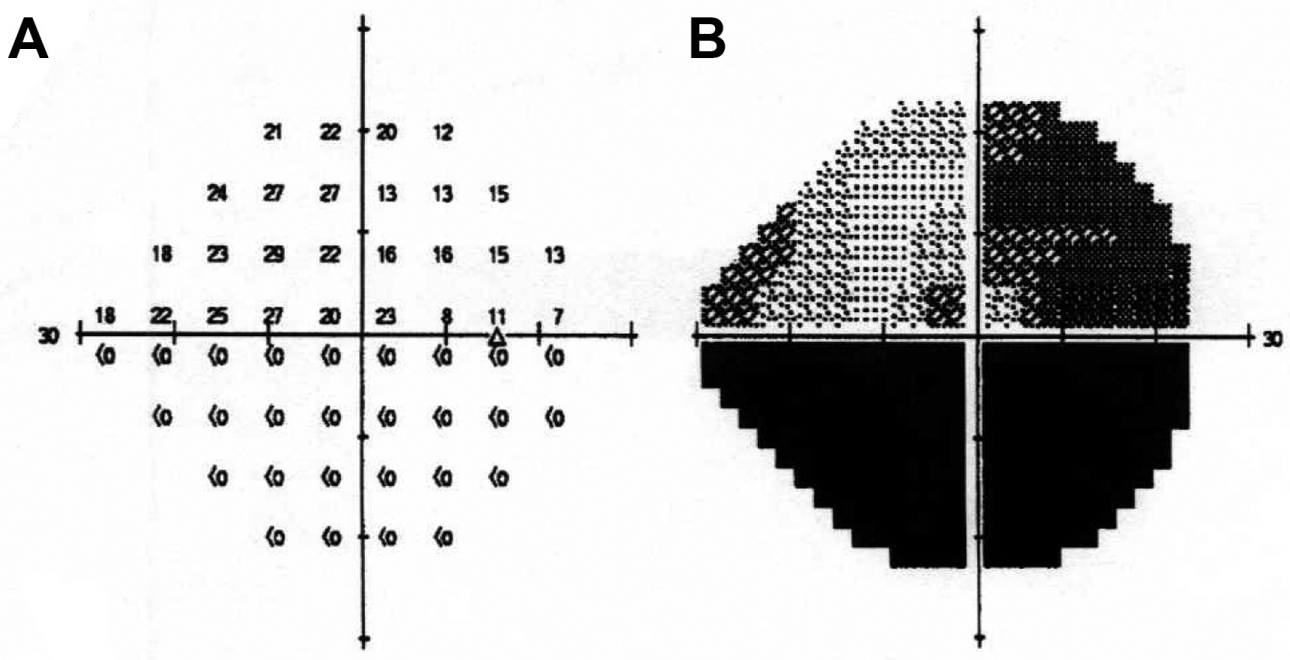

FIGURE 3: Automated perimetry using a Humphrey visual field 23 years post-radiosurgery.

Twenty-three years post-radiosurgery, a static, automated perimetry using a Humphrey visual field 24-2 study revealed a persistent, dense visual field loss with relative sparing of the superior nasal 


\section{Cureus}

quadrant. Fig. 3A represents the numeric $\mathrm{dB}$ graph and Fig. 3B, the gray-scale representation of Fig. $3 A$. The right eye $(O D)$ foveal sensitivity was 26 decibels $(\mathrm{dB})$ and the Median Deviation (MD) was $22.94 \mathrm{~dB}$. The OD visual acuity was $20 / 30$, after originally being light perception.
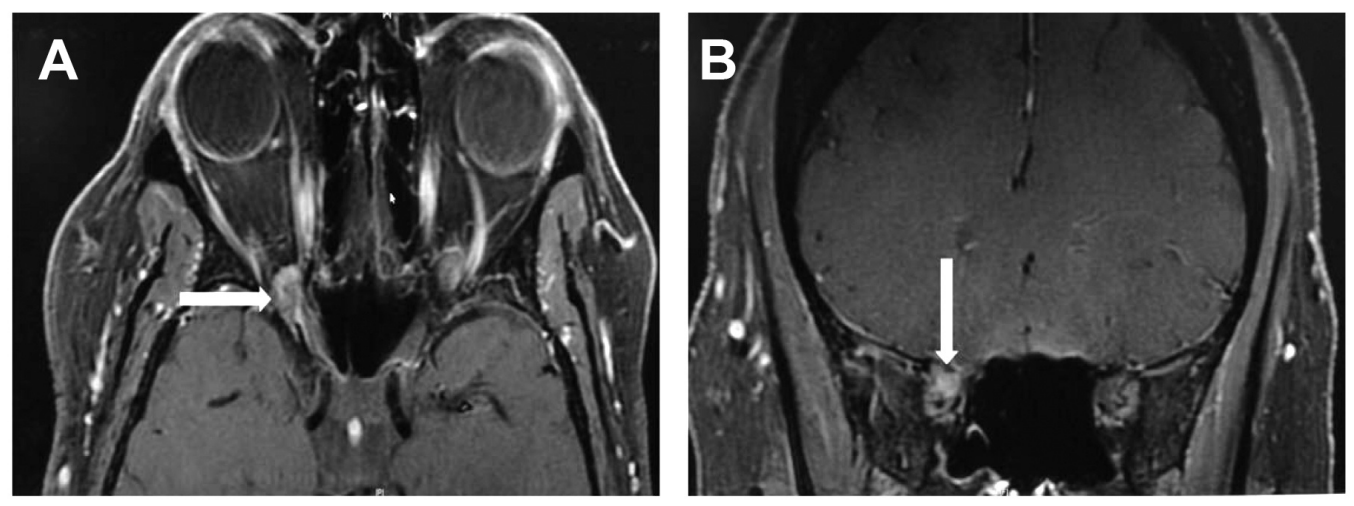

\section{FIGURE 4: Fat-suppressed contrast magnetic resonance imaging (MRI) of the orbits 23 years post-stereotactic radiosurgery.}

Fig. 4A is an axial image and Fig. 4B, a coronal slice, showing a stable, heterogeneously-enhancing small mass at the right orbital apex (arrows).

\section{Discussion}

Here, we describe the long-term follow-up of a patient who underwent high-dose SRS to a biopsy-confirmed residual ONSM. In the current modern era of meningioma radiosurgery, one would never use a dose anywhere near that used in the present case. However, a generation ago, the optimal dose for meningioma had yet to be defined, and a $20 \mathrm{~Gy}$ dose for lesions distant from the anterior visual pathways would have been considered unremarkable.

The exact dose delivered to the optic nerve in this case is hard to know, although the planned dose to the ONSM was $2500 \mathrm{cGy}$, as defined at the 80th percentile isodose, with an apparently intratumoral Dmax of 3125 cGy. Ideally, it might be possible to create a dose-volume histogram for the adjacent optic nerve, but that is not possible here with such small anatomic structures and the challenge of even visualizing the optic nerve within the orbital apex. However, given that nerve sheath meningiomas arise from the intraorbital meningoepithelium, i.e. the arachnoid cap cells located in an area between the arachnoid and the optic nerve's dural sheath which immediately abuts the optic nerve, a portion of the optic nerve must by definition have received close to the prescribed dose of $25 \mathrm{~Gy}$. Admittedly, the volume and length of nerve treated by this high dose of ionizing radiation was likely very small. Nevertheless, this very observation is itself consistent with the dose-volume effect, which is the empirically derived phenomenon that there exists a direct correlation between the amount (volume) of normal brain irradiated and the likelihood of radiation injury to that region of brain. In other words, when considering the risk factors underlying radiation injury, volume is a variable that is independent of radiation dose [3]. Of note, there is a least one other case report describing a patient with an ONSM treated with radiosurgery who experienced improved vision over eight years before losing all vision in the affected eye [4].

With specific respect to this case report, the smaller the volume of tissue, or perhaps alternatively here, the length of cranial nerve, the higher the dose tolerance of the irradiated structure. A related phenomenon has been reported in patients undergoing hypofractionated 
SRS for peri-optic lesions [5]. Regardless, to the best of our knowledge, this case report documents the highest dose of ionizing radiation given to any portion of the anterior visual pathway without apparent injury.

Are there alternative explanations to the witnessed results being described in this report? Although implausible, there is always the possibility that radiosurgery was inaccurately administered, and that this meningioma merely underwent a spontaneous involution. However, the timing of the patient's symptomatically improved vision correlates reasonably well with the known time frame for radiation; the patient's current 20/30 visual acuity in the OD is more than notable. Meanwhile, despite the good result after 23 years, it cannot be dismissed, out of hand, that this patient might not suffer ipsilateral visual compromise in the decades to come. However, ultimately, it is hard to escape the conclusion that in this single patient a portion of the affected optic nerve was heavily irradiated without any apparent deleterious effects over more than two decades of follow-up.

\section{Conclusions}

Although further research is clearly needed to better understand the limits of the dose-volume effect as it pertains to the anterior visual pathways, this individual patient experience emphasizes the role volume can possibly play in mediating the risk of visual injury in some patients undergoing radiosurgical ablation. By virtue of such, this observation could have potential utility in refining the radiosurgical approach among carefully selected patients.

\section{Additional Information \\ Disclosures}

Human subjects: Consent was obtained by all participants in this study. Stanford University 4947 issued approval 20863. Conflicts of interest: In compliance with the ICMJE uniform disclosure form, all authors declare the following: Payment/services info: All authors have declared that no financial support was received from any organization for the submitted work. Financial relationships: All authors have declared that they have no financial relationships at present or within the previous three years with any organizations that might have an interest in the submitted work. Other relationships: All authors have declared that there are no other relationships or activities that could appear to have influenced the submitted work.

\section{Acknowledgements}

Thanks to Beth Hoyte for her assistance in preparing figures for this paper, and of course, to my Cureus colleagues who inspire me each and every day!

\section{References}

1. Leavitt JA, Stafford SL, Link MJ, Pollock BE: Long-term evaluation of radiation-induced optic neuropathy after single-fraction stereotactic radiosurgery. Int J Radiat Oncol Biol Phys. 2013, 87:524-7. 10.1016/j.ijrobp.2013.06.2047

2. Chang SD, Adler JR, Jr: Treatment of cranial base meningiomas with linear accelerator radiosurgery. Neurosurg. 1997, 41:1019-25.

3. Flickinger JC, Lunsford LD, Kondziolka D: Dose-volume considerations in radiosurgery. Stereotact Funct Neurosurg. 1991, 51:99-105.

4. Kooshkabadi A, Ismayilov E, Kano H, Lunsford LD: Prolonged vision return after radiosurgery for an optic nerve-sheath meningioma. J Radiosurgery SBRT. 2012, 2:73-77.

5. Adler JR Jr, Gibbs IC, Puataweepong P, Chang SD: Visual field preservation after multisession CyberKnife radiosurgery for perioptic lesions. Neurosurg. 2008, 62:733-43.

10.1227/01.neu.0000316277.14748.63 